\title{
Analysis of gene expression and functional changes of adrenal gland in a rat model of kidney yang deficiency syndrome treated with Sini decoction
}

\author{
NONG TANG ${ }^{1}$, LIHONG LIU ${ }^{1}, \mathrm{HUA} \mathrm{QIU}^{2}, \mathrm{WEI} \mathrm{SHI}^{3}$ and DEWEN MAO ${ }^{2}$ \\ ${ }^{1}$ Department of Traditional Chinese Medicine, Guangxi University of Chinese Medicine; Departments of ${ }^{2}$ Hepatology and \\ ${ }^{3}$ Nephrology, The First Affiliated Hospital of Guangxi University of Chinese Medicine, \\ Nanning, Guangxi 530023, P.R. China
}

Received October 31, 2016; Accepted January 12, 2018

DOI: $10.3892 /$ etm.2018.6521

\begin{abstract}
Sini decoction (SND), a well-known traditional Chinese medicine, has been used to treat kidney Yang deficiency for $\sim 1,800$ years. The present study aimed to evaluate the effects of SND treatment on hypothalamic-pituitary-adrenal axis hormones in a rat model of Yang deficiency and to explore the molecular mechanisms using microarray analysis of adrenal glands and in vitro adrenocortical cell culture systems. The results indicated that SND treatment recovered circulating serum cortisol, adrenocortical hormone (ACTH) and testosterone levels in a yang deficiency model. Immunohistochemical analysis of pituitary and hypothalamic tissues confirmed increased expression of ACTH and corticotropin-releasing factor, respectively, in response to SND treatment. Microarray analysis identified a marked upregulation of genes involved in $\leq$ metabolic and stress response pathways in rat adrenal tissues in response to SND treatment, exemplified by cyclooxygenase- 2 and nuclear factor $(\mathrm{NF})-\kappa \mathrm{B}$. In vitro, SND exerted a protective effect on mitochondria in response to $\mathrm{H}_{2} \mathrm{O}_{2}$ exposure also activated $\mathrm{NF}-\kappa \mathrm{B}$ and cyclic adenosine monophosphate response element binding protein reporter gene activity. These results contributed towards an improved understanding of how SND effectively alleviates the symptoms of kidney Yang deficiency syndrome at the molecular level.
\end{abstract}

Correspondence to: Professor Dewen Mao or Dr Hua Qiu, Department of Hepatology, The First Affiliated Hospital of Guangxi University of Chinese Medicine, 89-9 Dongge Road, Nanning, Guangxi 530023, P.R. China

E-mail: mdwboshi2005@sina.com

E-mail: qiuhua8899@163.com

Key words: Sini decoction, kidney, yang deficiency, cyclooxygenase-2

\section{Introduction}

In contrast to target-based Western medicine, Traditional Chinese Medicine (TCM) employs a conceptual framework whereby the body's balance of Yin-Yang energy is a crucial factor in controlling normal functioning or homeostasis. According to the concepts of TCM, Yang represents warmth and promotion of beneficial functions, whilst Yin represents suppression of nourishment. Kidney Yang deficiency syndrome (KDS-Yang) is one of the classical syndrome patterns in TCM and is characterized by disorders of multiple metabolic pathways. Modern research has indicated that functional disorders and damage to the hypothalamic-pituitary-adrenal (HPA) axis are the major pathological mechanisms underlying KDS-Yang (1), with the key issue being deregulated expression of adrenocortical hormone (ACTH) $(2,3)$. Patients with KDS-Yang invariably present with symptoms including pain and weakness of the waist and knees, feeling cold, fatigue, impaired hearing and tooth loss, and this syndrome is often observed in the later stages of several common chronic diseases, including rheumatoid arthritis, diabetes and hypertension (4-6).

Sini decoction (SND) is a well-known remedy in TCM that has been used to restore Yang body energy and treat KDS-Yang and associated diseases for $\sim 1,800$ years $(7,8)$. In recent years, advances in metabonomics have enabled identification of metabolic signatures of KDS-Yang $(9,10)$ and therapeutic biomarkers of SND $(11,12)$. Despite this, the sites of action, molecular targets and active components of SND have remained largely elusive.

The present study analyzed the in vivo effects of SND treatment on HPA-axis circulating and in situ hormone levels in a rat model of Yang deficiency. Of note, gene microarray analysis of rat adrenal gland tissues identified a marked upregulation of genes involved in stress and metabolic response pathways as a consequence of SND treatment. Furthermore, the present study highlighted a role for SND in regulating the expression of cyclooxygenase (COX-2), also known as prostaglandin-endoperoxide synthase 2 (PTGS2), in adrenocortical cells, reflecting the potential of SND treatment to control prostaglandin release and contribute to balanced sodium and 
water homeostasis, possibly through nuclear factor (NF)- $\mathrm{B}$ or cyclic adenosine monophosphate (cAMP)-mediated signalling. Of note, the results also suggested that SND, either directly or indirectly through COX-2 and prostaglandin synthesis, protects against oxidative cellular damage caused by reactive oxygen species (ROS).

\section{Materials and methods}

Reagents. Hydrocortisone injection solution was purchased from Tianjin Jiaozuo Pharmaceutical Co. (Tianjin, China). ELISA kits for detection of testosterone, ACTH and cortisol (CORT) were obtained from Elabscience (cat. no. E-EL-0072c, E-EL-R0048c and E-EL-R0269c, respectively). 2'-7'-Dichlorodihydrofluorescein diacetate (DCFH-DA) was from Sigma-Aldrich (Merck KGaA, Darmstadt, Germany). The NCI-H295R (H295R) cell line was obtained from the American Type Culture Collection (ATCC; no. CRL-2128 ${ }^{\mathrm{TM}}$ ). Dulbecco's modified Eagle's medium (DMEM) was from Gibco (Thermo Fisher Scientific, Inc., Waltham, MA, USA) and fetal bovine serum (FBS) was from Biowest (Nuaillé, France). The mitochondrial membrane potential detection kit (cat. no. C2006) was from Beyotime Institute of Biotechnology (Haimen, China).

Preparation of SND. According to the original composition of SND recorded in the Chinese Pharmacopoeia 2010 edition (13), SND was prepared using the following procedure: The crude drugs of Acontium carmichaeli (90 g), Zingiber officinale (60 g) and Glycyrrhiza uralensis (90 g) were immersed in 2.4 1 water for $1 \mathrm{~h}$ and then decocted to boil for $2 \mathrm{~h}$. The decoction was filtered through four layers of gauze. Next, the drugs were boiled once again for $1 \mathrm{~h}$ with $1.9 \mathrm{l}$ water and the decoction was filtered again using the abovementioned method. The successive decoctions were merged and condensed under decompression. Finally, the extracted solution was made up to a concentration of $1.0 \mathrm{~g}$ crude $\mathrm{drug} / \mathrm{ml}$.

Animal experiment. Animal experiments were performed in accordance with the Guidelines for Animal Experimentation of Guangxi University of Chinese Medicine (Nanning, China). The protocols were approved by the ethics committee of the First Affiliated Hospital of Guangxi University of Chinese Medicine (Nanning, China). A total of 30 Male Wistar rats (age, 9 weeks; body weight, 200-250 g, Shanghai SLAC Laboratory Animal Co., Ltd., Shanghai, China) were kept under standard conditions with regulated temperature $\left(17-25^{\circ} \mathrm{C}\right)$ and relative humidity (45-60\%) under a 12-h light/dark cycle. The animals had ad libitum access to food and drinking water throughout the study period. After one week of habituation, rats were randomly assigned to 5 groups: Blank, Model, SND-low dose, SND-high dose and Saline, as depicted in the schematic in Fig. 1A. In the SND treatment groups, rats were intraperitoneally (i.p.) injected with hydrocortisone at a dose of $10 \mathrm{mg} / \mathrm{kg}$ once daily for 15 days and then administered SND extract by oral gavage twice daily for 15 days (high dose, $6.3 \mathrm{~g}$ decoction $/ \mathrm{kg}$ body weight; low dose, $1.26 \mathrm{~g}$ decoction $/ \mathrm{kg}$ body weight). In the Saline group, rats were intraperitoneally injected with hydrocortisone at a dose of $10 \mathrm{mg} / \mathrm{kg}$ once daily for 15 days and then orally administered normal saline twice daily for 15 days. Rats in the Model group received 15 days +15 days of hydrocortisone (i.p.) at a dose of $10 \mathrm{mg} / \mathrm{kg}$ once daily (9). Rats in the Blank group received 15 days of saline (i.p.), followed by 15 days of oral saline (Fig. 1A). On Days 0 , 15 and $30,400 \mu 1$ of blood from tail vein of each rat were collected into heparinized tubes and immediately centrifuged at $14,360 \mathrm{x}$ g for $10 \mathrm{~min}$. The plasma was transferred into clean tubes and stored at $-80^{\circ} \mathrm{C}$ until biochemical analysis.

Immunohistochemistry. Immunohistochemistry was performed on a $4 \mu \mathrm{m}$ thick formalin-fixed (immersed in $10 \%$ formalin for $24 \mathrm{~h}$ at room temperature) paraffin-embedded sections using the Real EnVision Detection System and Peroxidase/diaminobenzidine (Dako; Agilent Technologies, Inc., Santa Clara, CA, USA) according to the manufacturer's protocol with minor modifications. Briefly, tissue sections were deparaffinized, and rehydrated using graded alcohols. Antigen retrieval was performed by heating the slides in a microwave oven $(500 \mathrm{~W}$ for 5 min 3 times in citrate buffer $\mathrm{pH} 6.0$ at $96^{\circ} \mathrm{C}$ ) (14). Endogenous peroxidase was quenched for $10 \mathrm{~min}$ with peroxidase blocking reagent. The sections were washed using PBS and incubated in blocking solution containing $2 \%$ donkey serum (cat. no. AR009; Boster Biological Technology, Pleasanton, CA, USA), followed by incubation overnight at $2-4^{\circ} \mathrm{C}$ with primary antibodies [corticotropin-releasing hormone (CRH) antibody, cat. no. BA1503; 1:100; ACTH antibody, cat. no. BA0003-1; 1:100; Boster Biological Technology]. Following washing with PBS buffer, sections were incubated with peroxidase labelled secondary antibodies from the Envision kit for $40 \mathrm{~min}$ at room temperature prior to staining with diaminobenzidine substrate chromogen (also sourced from the kit) for $10 \mathrm{~min}$ at room temperature. Images were recorded using confocal microscopy (Leica Microsystems GmbH, Wetzlar, Germany). Cells with dark staining were counted as positive and analysed using Image-Pro ${ }^{\circledR}$ Plus version 5.1 (Media Cybernetics, Inc., Rockville, MD, USA) software.

Cell culture and assays. The H295R cell line was cultured in DMEM medium containing 10\% FBS. For the 2'-7'-dichlorodihydrofluorescein diacetate (DCFH-DA) assay, cells were cultured in 96-well plates and labeled with DCFH-DA $(10 \mu \mathrm{M})$ or unlabeled (none) and then cultured for an additional $3 \mathrm{~h}$. Cells were then analyzed on a fluorescence reader using excitation/emission wavelengths of 485/535 nm. For the JC-1 assay, cells were cultured in 96-well plates and stained with JC-1 according to the manufacturer's instructions. Fluorescence of J-aggregates were measured on the plate reader using excitation/emission wavelengths of $535 / 595 \mathrm{~nm}$. In the assay that suppressed the upregulation of COX-2, H295R cells were treated in vitro with either vehicle control (blank group), SND alone, $\mathrm{H}_{2} \mathrm{O}_{2}$ for $30 \mathrm{~min}\left(\mathrm{H}_{2} \mathrm{O}_{2}\right.$ group) or SND pre-treatment for $24 \mathrm{~h}$ followed by $\mathrm{H}_{2} \mathrm{O}_{2}$ for a further $30 \mathrm{~min}$ ( $\mathrm{SND}+\mathrm{H}_{2} \mathrm{O}_{2}$ group). Cells were then collected for quantitative real-time polymerase chain reaction (PCR).

Gene microarray analysis. To further investigate the potential modes of action of SND treatment, a gene microarray analysis of mRNA derived from rat adrenal gland tissues from the blank, control and SND treatment groups was performed at day 30 (Fig. 1A). mRNA was isolated and analyzed using a 
A

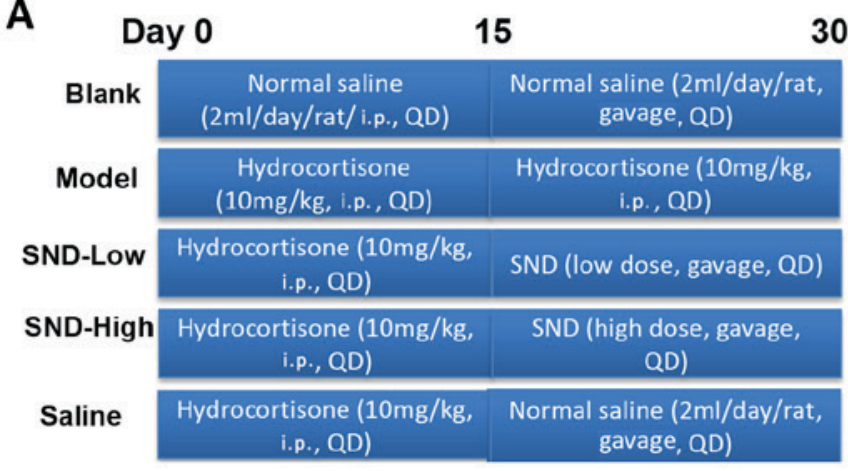

C

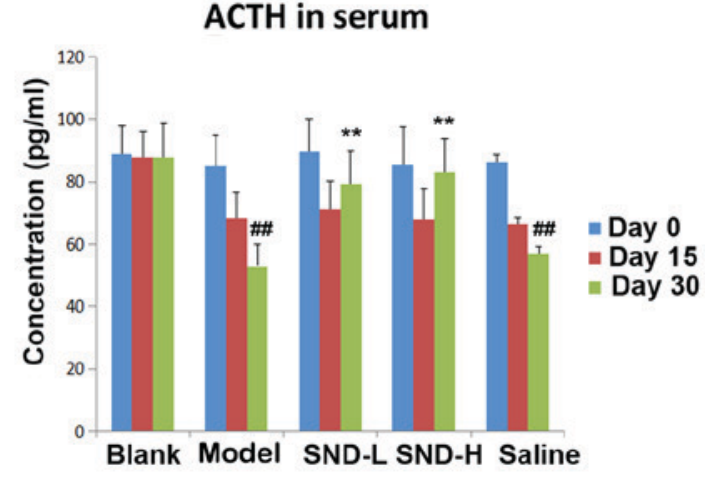

$30 \mathrm{~B}$

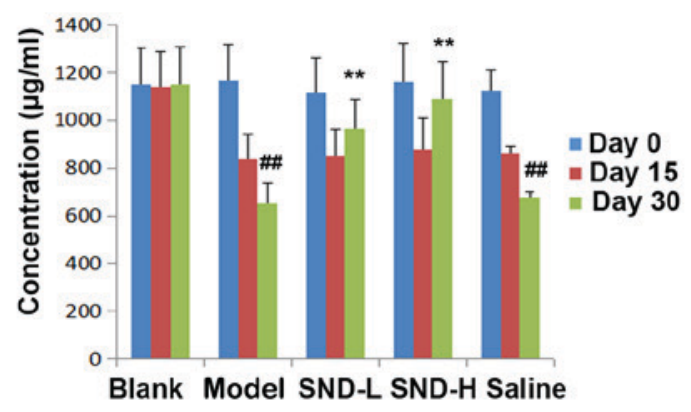

D

Testosterone in serum

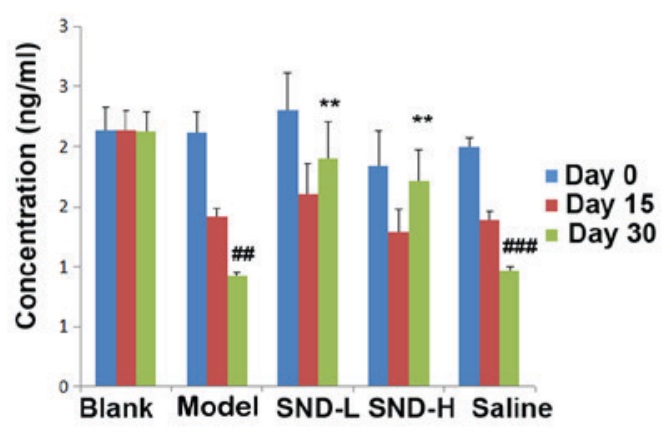

Figure 1. SND ameliorates Yang deficiency in a rat model. (A) Scheme illustrating the study design. Rats were grouped (n=6/group) and treated as indicated in for 30 days. Sera were collected at day 0, 15 and 30 and subjected to ELISA detection of (B) CORT, (C) ACTH, and (D) testosterone. ${ }^{\#}$ P $<0.01$ vs. blank group at day $30 .{ }^{* *} \mathrm{P}<0.01$ vs. saline group. CORT, cortisol; SND-L, SND-low dose; SND-H, SND-high dose; SND, Sini decoction; ACTH, adrenocortical hormone.

rat-specific Affymetrix GeneChip 1.0 ST Array (Thermo Fisher Scientific, Inc., Waltham, MA, USA). The cluster algorithm test series of gene expression dynamics was used to profile the gene expression series and to identify the most probable set of clusters generating the observed series. Gene ontology (GO) analysis was applied to analyze the main function of differentially expressed genes according to GO database and the AmiGO database (http://geneontology.org/; http://amigo. geneontology.org/amigo). Pathway analysis was used to identify significant pathways of the differentially expressed genes according to the Kyoto Encyclopedia of Genes and Genomes database. Data were analyzed using GO analysis software. Comparisons were made between control vs. blank, low-dose SND vs. control and high-dose SND vs. control. Genes that were significant in the GO as well as the pathway analyses were identified. Differential gene expression analyses were performed using the criteria of +2 -fold or -0.5 -fold differential expression and $\mathrm{P}<0.05$.

Reverse transcription (RT) quantitative ( $q$ ) PCR. RNA from H295R cells was extracted using the RNeasy kit (Qiagen Inc., Valencia, CA, USA) according to the manufacturer's protocol. cDNA was synthesized, and quantitative RT-PCR analysis was performed using a Bio-Rad CFX 96 cycler (Bio-Rad Laboratories Inc., Hercules, CA, USA) with the SuperScript III two-step RT-qPCR kit with SYBR Green (Invitrogen; Thermo Fisher Scientific, Inc.). The primer sequences utilized were as follows: COX-2 forward, 5'-AGCCAGGCAGCAAATCCTT-3' and reverse, 5'-GGGTGGGCTTCAGCAGTAAT-3'; Actin (used as the reference gene) forward, 5'-AGAGGGAAATCG TGCGTGAC-3' and reverse, 5'-CCATACCCAAGAAGGAAG
GCT-3'. The thermocycling conditions were as follows: Initial denaturation at $95^{\circ} \mathrm{C}$ for $1 \mathrm{~min}$ followed by 39 cycles at $95^{\circ} \mathrm{C}$ for $10 \mathrm{sec}, 65^{\circ} \mathrm{C}$ for $30 \mathrm{sec}$, and $72^{\circ} \mathrm{C}$ for $30 \mathrm{sec}$. The relative expression analysis of target genes were performed using the $2^{-\Delta \Delta \mathrm{Cq}}$ method $(15,16)$.

Reporter assays. H295R cells (seeded at $10^{5}$ cells/well in 24-well plates) were co-transduced with $\mathrm{NF}-\kappa \mathrm{B} /$ renilla lentiviral particles and CRE/renilla lentiviral particles. $\mathrm{NF}-\kappa \mathrm{B}, \mathrm{CRE}$ and renilla lentiviral particles were obtained from Novobio Scientific, Inc. (Shanghai, China). The NF- $\kappa$ B reporter lenviral vector contained an $\mathrm{NF}-\kappa \mathrm{B}$ responsive element (17), while the CRE reporter vector contained a cAMP response-binding element (18). The Renilla lentiviral vector expressing renilla luciferase was used as an internal control. Following transduction at a multiplicity of infection of 50 for $8 \mathrm{~h}$, blasticidin ( $4 \mu \mathrm{g} / \mathrm{ml}$; Invitrogen; Thermo Fisher Scientific, Inc.) were then added to screen stably transduced cells for 2 weeks. H295R cells expressing NF- $\kappa \mathrm{B} /$ renilla reporters were subjected to SND treatment $(50 \mu \mathrm{g} / \mathrm{ml})$ for $48 \mathrm{~h}$, while CRE/renilla expressing cells were treated with forskolin $(5 \mu \mathrm{M})$ or forskolin $(5 \mu \mathrm{M})+\mathrm{SND}(50 \mu \mathrm{g} / \mathrm{ml})$ for $48 \mathrm{~h}$. Cells were collected and luciferase assays were performed using a luminescence plate reader and Dual-Luciferase Reporter Assay (cat. no. E1910; Promega Corporation, Madison, WI, USA). The relative luciferase activity for each sample was calculated as a ratio of firefly luciferase activity divided by renilla luciferase activity.

Statistical analysis. Data were analyzed using GraphPad Prism software version 6.0 (GraphPad Inc., La Jolla, CA, USA) and 
values are expressed as the mean \pm standard error of the mean. The significance of differences was assessed using either a two-tailed, non-paired Student's t-test or a one-way ANOVA and statistical significance was defined using the customary threshold of $\mathrm{P}<0.05$ (95\% confidence interval).

\section{Results}

Treatment with SND ameliorates KDS-Yang in a rat model. In order to assess the effects of SND treatment on the HPA axis, a rat model of Yang deficiency was established by treating animals daily with high-dose hydrocortisone $(10 \mathrm{mg} / \mathrm{kg}$, i.p.) for 15 days, followed by withdrawal (8). At this point, rats were divided into four groups (six rats in each group) and treated with either continued hydrocortisone $(10 \mathrm{mg} / \mathrm{kg}$, i.p. Model group), low-dose oral SND (SND-Low), high-dose oral SND (SND-High) or normal saline (Saline) for a further 15 days. The Blank group received i.p. saline injection for 15 days ( $2 \mathrm{ml} /$ day), followed by oral saline for 15 days $(2 \mathrm{ml} /$ day $)$ (Fig. 1A). Sera were collected from all rats on days 0,15 and 30 and subjected to ELISA to measure the levels of CORT, ACTH and testosterone. As expected, KDS-Yang model animals exhibited a significant decline in circulating cortisol levels, with a more pronounced decrease observed with longer hydrocortisone treatment $(\mathrm{P}<0.01$; Fig. 1B). Of note, animals in the low- and high-dose SND groups exhibited a marked recovery of circulating CORT as compared with the saline group on day 30 ( $\mathrm{P}<0.01$; Fig. 1B). Similarly, KDS-Yang model group also displayed a significant decline in circulating ACTH and testosterone levels (Fig. 1C and D), with a further decline observed with increasing duration of hydrocortisone treatment $(\mathrm{P}<0.01)$. After 15 days of hydrocortisone treatment, 15 days of either low- or high-dose SND were sufficient for recovery of the circulating ACTH and testosterone levels, when compared with those in the group treated with normal saline for 15 days after hydrocortisone withdrawal $(\mathrm{P}<0.01$; Fig. $1 \mathrm{C}$ and $\mathrm{D})$.

To further explore the mechanism underlying the attenuation of HPA-axis hormone decline by SND treatment, rat pituitary gland and hypothalamus tissues were obtained from each treatment group at day 30. Tissue sections were subjected to immunohistochemical analysis to detect the expression of $\mathrm{ACTH}$ and $\mathrm{CRH}$ proteins in the pituitary gland and hypothalamus tissues, respectively. Three slides from each rat were analysed and results were quantified from these images. Representative images are presented Fig. 2A. Relative to the saline-treated control group, KDS-Yang rats exhibited a marked decline in pituitary ACTH levels (Fig. 2A and B). Treatment with low-dose SND produced a marginal elevation in ACTH levels and high-dose SND resulted in a significant increase in pituitary ACTH levels to exceed those in the saline and KDS-Yang controls (Fig. 2A and B). The levels of $\mathrm{CRH}$ in the hypothalamus also exhibited a reduced trend in the KDS-Yang rats (Fig. 2A and C). Of note, this trend was reversed upon high-dose SND treatment, while low-dose SND treatment had no effect (Fig. 2C).

Genes involved in metabolic and stress response pathways are upregulated in the adrenal gland in response to SND treatment. To further investigate the potential modes of action of SND treatment, a gene microarray analysis of mRNA derived from adrenal gland tissues obtained from treated rats at 30 days was performed. mRNA was isolated and analyzed using a rat-specific Affymetrix GeneChip 1.0 ST Array. Data were analyzed using GO analysis software and comparisons of control vs. blank, low-dose SND vs. control and high-dose SND vs. control were made. Differential gene expression analyses demonstrated that adrenal gland tissues from SND-treated (high-dose) vs. control-treated rats displayed marked differences in gene expression. Those genes with the highest differential expression scores included $\mathrm{COX}-2, \mathrm{NF}-\kappa \mathrm{B}$ and genes involved in cAMP-dependent signalling. Genes were clustered into categories based on the GO system and ordered according to the highest enrichment (Fig. 3A). GO analysis was used to assign these GO terms to kyto encyclopaedia of genes and genome pathways and again, these pathways were ordered according to the highest enrichment (Fig. 3B). According to these analyses, differentially expressed genes in the SND-treated (high-dose) vs. control-treated rat adrenal glands were enriched in transcriptional regulation and metabolic signaling.

SND treatment results in partial suppression of $\mathrm{COX}-2$ upregulation in response to $\mathrm{H}_{2} \mathrm{O}_{2}$ and protects adrenocortical cells from ROS in vitro. As SND treatment resulted in significant upregulation of genes predominantly involved in metabolic and stress response signaling, the present study sought to further confirm and explore the consequences of this in vitro using the adrenocortical cell line H295R. Due to the known roles of $\mathrm{COX}-2$ in regulating the response to kidney ischemia/reperfusion injury, the effects of SND pre-treatment on COX-2 expression following $\mathrm{H}_{2} \mathrm{O}_{2}$ treatment were assessed. COX-2 mRNA expression was measured by reverse transcription-quantitative polymerase chain reaction analysis. H295R cells were treated in vitro with either vehicle control (blank group), SND alone, $\mathrm{H}_{2} \mathrm{O}_{2}$ for 30 min $\left(\mathrm{H}_{2} \mathrm{O}_{2}\right.$ group) or SND pre-treatment for $24 \mathrm{~h}$ followed by $\mathrm{H}_{2} \mathrm{O}_{2}$ for a further $30 \mathrm{~min}$ (SND $+\mathrm{H}_{2} \mathrm{O}_{2}$ group) (Fig. 4A). Treatment with $\mathrm{H}_{2} \mathrm{O}_{2}$ resulted in a clear induction of COX-2 gene expression compared with that in the vehicle-treated group ( 15-fold induction) or SND-pre-treated blank group ( $\mathrm{P}<0.01$; Fig. 4A). Of note, pre-treatment of cells with SND for $24 \mathrm{~h}$ resulted in a significant suppression of COX-2 induction compared with that in the group treated with $\mathrm{H}_{2} \mathrm{O}_{2}$ only.

To further assess the functional consequences of this SND-mediated partial suppression of COX-2 expression, the effects of pre-treatment with SND on the $\mathrm{H}_{2} \mathrm{O}_{2}$-induced generation of ROS were determined using the cell permeable dye DCFH-DA. In the presence of intracellular ROS, DCFH-DA is rapidly oxidized to fluorescent DCF. As displayed in Fig. 4B, treatment with $\mathrm{H}_{2} \mathrm{O}_{2}$ for 15 min resulted in a significant elevation of ROS above the background (blank) levels. However, pre-treatment of the cells with SND $24 \mathrm{~h}$ followed by $\mathrm{H}_{2} \mathrm{O}_{2}$ treatment did not exhibit elevation, indicating that ROS generation was prevented by SND.

As one of the major consequences of ROS-induced damage is destruction of mitochondria through loss of the mitochondrial membrane potential, ultimately leading to cellular apoptosis, the ability of SND to protect against ROS-induced loss of mitochondrial membrane potential was then investigated. 

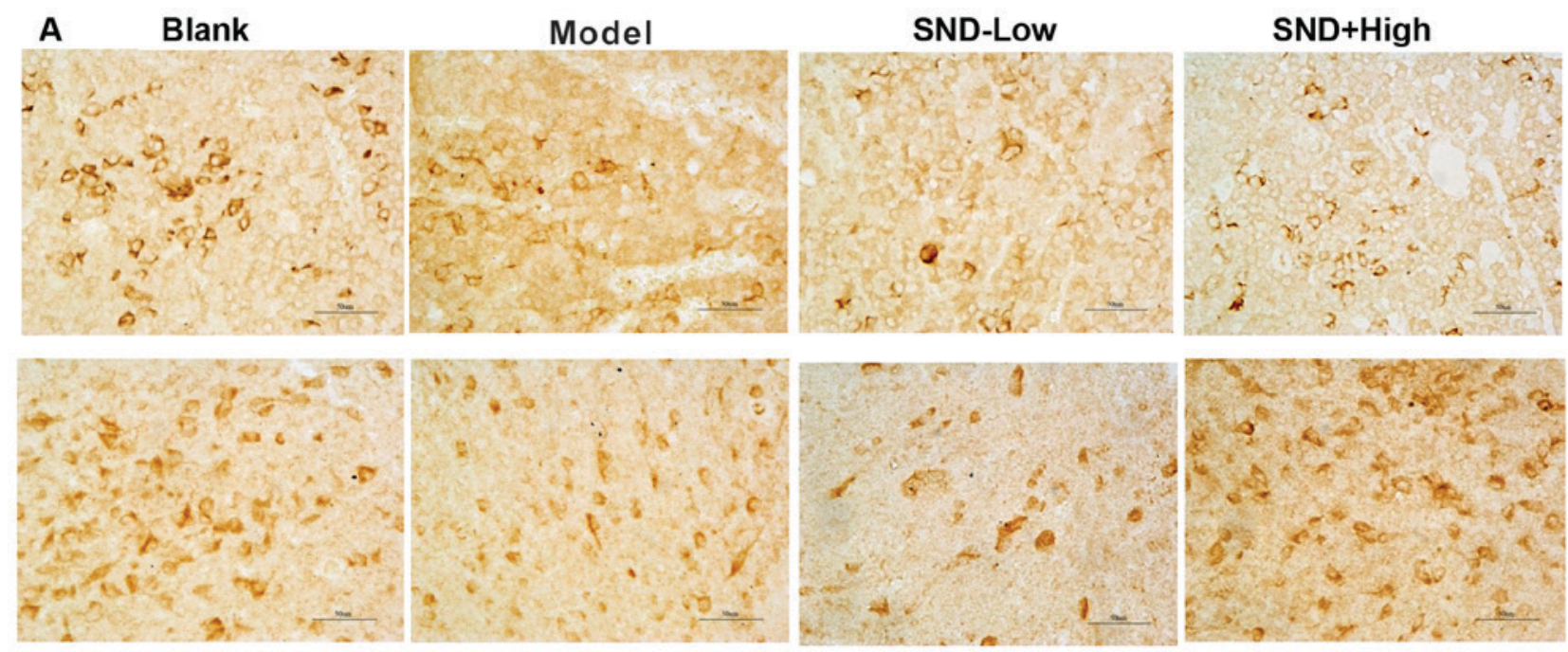

B

ACTH-Pituitary

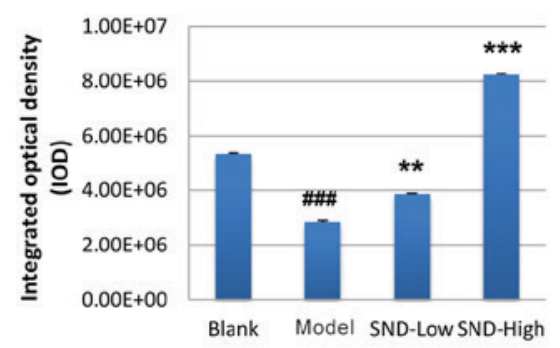

C CRF-Hypothalamus

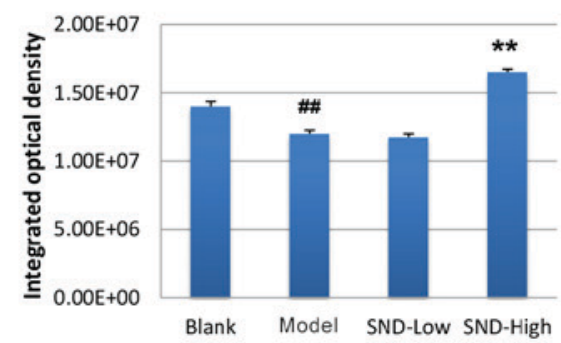

Figure 2. (A) SND treatment reverses the hydrocortisone-induced suppression of pituitary ACTH and hypothalamic CRF as measured using immunohistochemistry. Images are representative of three tissue slices (magnification, x40; scale bars, $50 \mu \mathrm{m}$ ). Quantified data of pituitary (B) ACTH and (C) hypothalamic

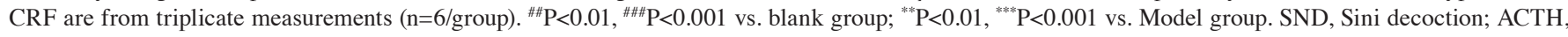
adrenocortical hormone; $\mathrm{CRF}$, corticotropin-releasing factor.

For this, H295R cells subjected to the above treatments were stained with the membrane-permeant dye JC-1. JC- 1 is widely used in apoptosis studies to monitor mitochondrial health. Depending on the mitochondrial membrane potential, the JC-1 dye accumulates in mitochondria, which is indicated by a fluorescence emission shift from green $(\sim 529 \mathrm{~nm})$ to red $(\sim 590 \mathrm{~nm})$. Consequently, mitochondrial depolarization is indicated by a decrease in the red/green fluorescence intensity ratio. The potential-sensitive color shift is due to the concentration-dependent formation of red fluorescent J-aggregates. Vehicle-treated (blank) H295R cells displayed the expected accumulation of red JC-1 dye within the mitochondria, whilst $\mathrm{H}_{2} \mathrm{O}_{2}$-treated cells exhibited a significant decrease in the level of red JC-1 fluorescence due to ROS-induced damage and loss of mitochondrial membrane potential (Fig. 4C). However, pre-treatment of $\mathrm{H} 295 \mathrm{R}$ cells with SND prior by $\mathrm{H}_{2} \mathrm{O}_{2}$ treatment increased the level of JC-1-associated red staining to a level similar to that in the vehicle (control) group, indicating that SND maintained the mitochondrial membrane potential and presumably prevented mitochondrial membrane damage.

SND treatment of $H 295 R$ cells results in transcriptional activation through $N F-\kappa B$ and $c A M P$ response-binding element $(C R E)$-binding proteins in vitro. Based on known roles of $N F-\kappa B$ and $C R E$ transcription factor activity in regulating COX-2 gene expression and previous studies on Yang deficiency (19-22), the present study sought to explore whether in vitro SND treatment resulted in activation of $\mathrm{NF}-\kappa \mathrm{B}$ or CRE activity in H295R cells. To assess this, stably transfected $\mathrm{H} 295 \mathrm{R}$ cells were generated expressing lentiviral $\mathrm{NF}-\kappa \mathrm{B}$ or CRE-responsive luciferase reporter constructs and subjected to SND treatment for $24 \mathrm{~h}$. Reporter activity was assessed using a luminescence plate reader. Treatment of H295R cells with SND resulted in a significant increase in NF- $\kappa \mathrm{B}$-driven luciferase activity compared with that in the vehicle-treated (blank) controls ( 8,300 vs. $\sim 6,900$ units; Fig. 5A). With regard to CRE-driven luciferase activity, when the adenylyl cyclase activator forskolin was used to artificially elevate intracellular cAMP levels, SND treatment enhanced the effect compared with that of forskolin alone ( 2,500 vs. 2,000 units; Fig. 5B).

\section{Discussion}

Recovery of body Yang energy using SND is well known in TCM, but the underlying molecular mechanism has remained to be elucidated. Using an in vivo rat model of KDS-Yang, the present study demonstrated multiple effects of SND treatment on the HPA-axis, including restoration of circulating serum levels of CRH, ACTH and CORT, and importantly, an elevation of in situ expression of ACTH. Based on prior literature describing the ability of SND to improve adrenal gland function (23), the present study focused on investigating the effects 
A

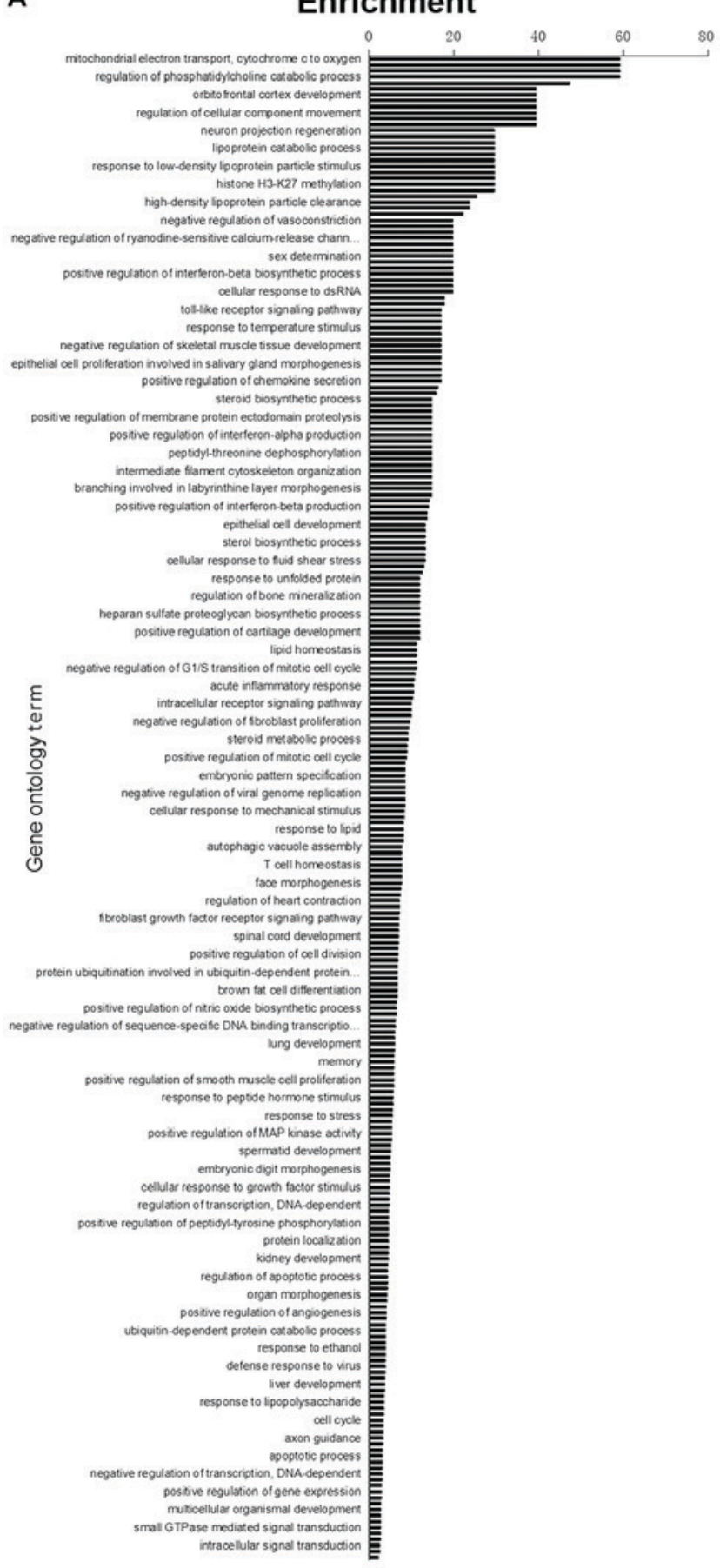

B

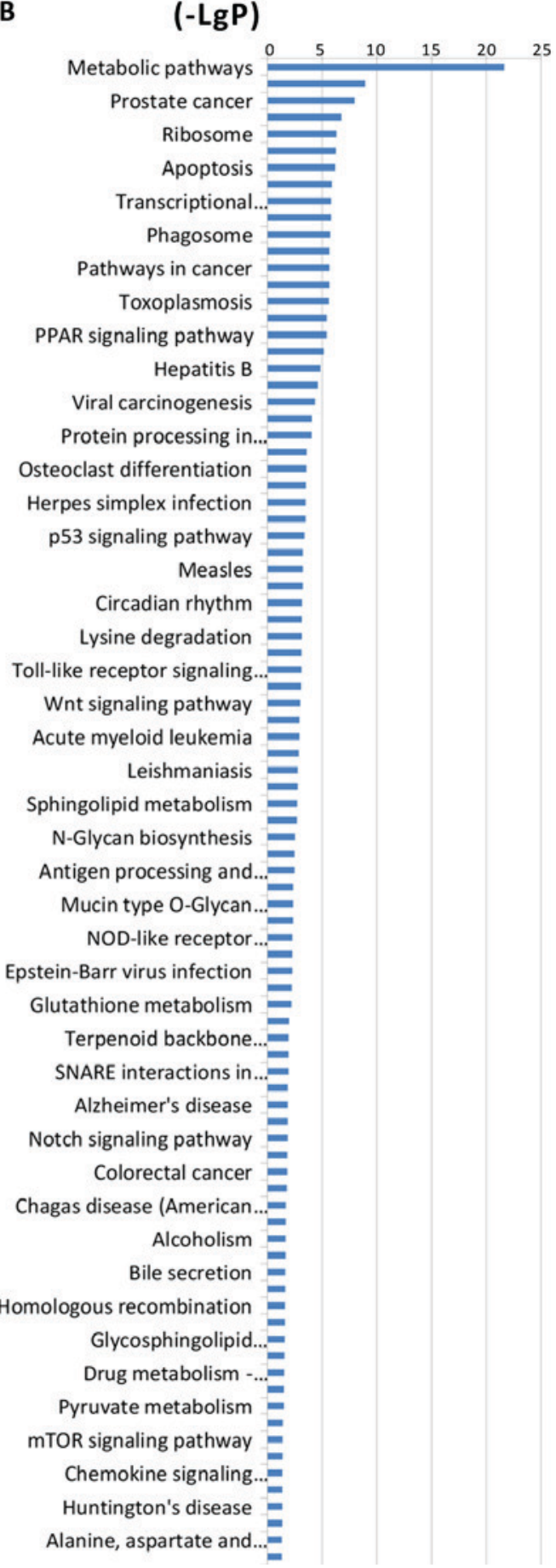

Figure 3. Microarray analysis results of rat adrenal gland tissue samples. Adrenal gland tissue samples were prepared and analyzed. Values are expressed as the mean \pm standard error of the mean ( $\mathrm{n}=6 /$ group). (A) Enrichment values for different gene ontology terms; (B) (-LgP) values for different signaling pathways. If $\mathrm{P}$-values are the same, a higher enrichment value indicates a higher impact of experiments on associated genes. A higher (-LgP) value indicates a lower P-value, and the significance is higher. MAP, mitogen-activated protein; PPAR, peroxisome proliferator-activated receptor; NOD, nucleotide-binding oligomerization domain; SNARE, Soluble NSF Attachment Protein receptor.

of SND treatment at the molecular level using gene microarray analysis of rat adrenal glands. To the best of our knowledge, the present study was the first to pursue this approach of studying the mechanisms of the biological effects of SND, and a variety of results were obtained, several of which indicated the restoration of HPA-axis functionality. Of note, a marked upregulation of genes implicated in metabolic pathway activation and stress responses were observed, including $\mathrm{COX}-2$ and $\mathrm{NF}-\kappa \mathrm{B}$. In the context of the KDS-Yang model, in which the HPA-axis activity is repressed as a consequence of prolonged high-dose glucocorticoid treatment, induction of COX-2 expression by SND is expected to reactivate HPA-axis activity due to the ability of COX-2 to drive proliferative, pro-inflammatory and injury responses (24). Within the kidney, COX-2 has known roles in regulating renal physiology through prostaglandin synthesis, control of fluid excretion and intrarenal hemodynamics (25), thus underscoring the importance of COX-2 expression in controlling renal homeostasis. 
A

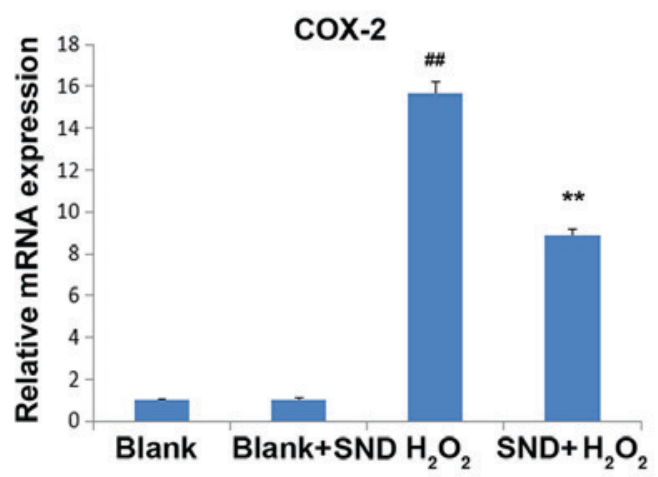

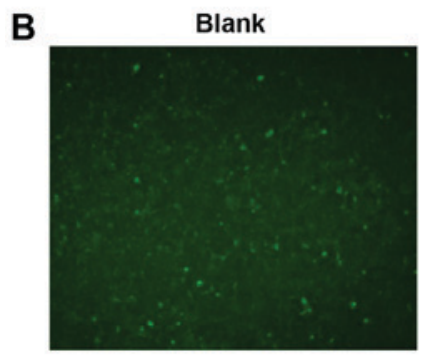
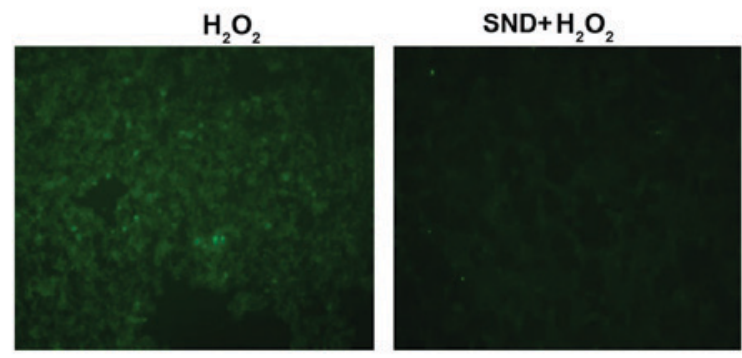

C
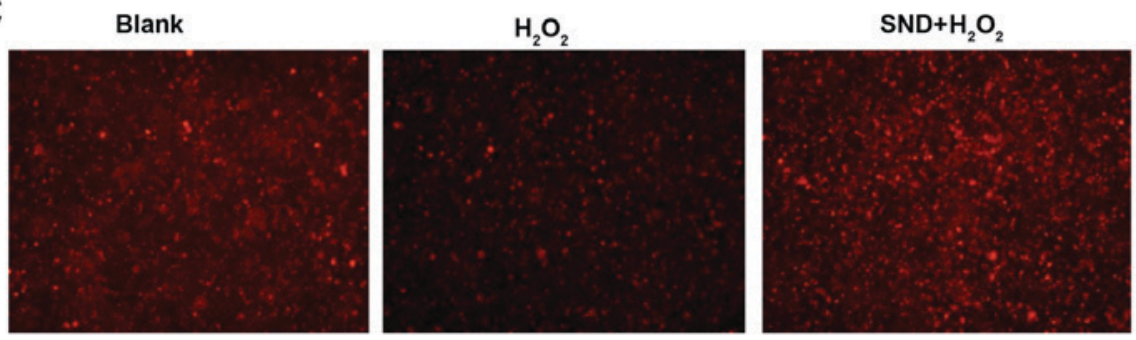
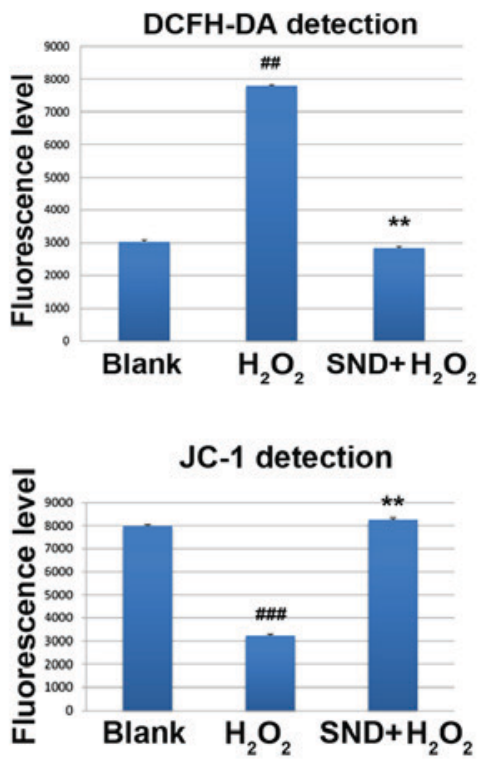

Figure 4. (A) SND treatment exerts anti-oxidant effects in $\mathrm{H} 295 \mathrm{R}$ cells. Adrenocortical H295R cells were subjected to pre-treatment with or without SND for $24 \mathrm{~h}$, followed by incubation with $\mathrm{H}_{2} \mathrm{O}_{2}$ for $30 \mathrm{~min}$. In all groups, culture media was replaced at $24 \mathrm{~h}$ following treatment and COX-2 mRNA expression was measured. (B) SND treatment antagonizes $\mathrm{H}_{2} \mathrm{O}_{2}$-induced generation of ROS in adrenocortical $\mathrm{H} 295 \mathrm{R}$ cells. $\mathrm{H} 295 \mathrm{R}$ cells were subjected to pre-treatment with or without SND for $24 \mathrm{~h}$, followed by $\mathrm{H}_{2} \mathrm{O}_{2}$ treatment for $15 \mathrm{~min}$. ROS generation evaluated by quantification of DFCH-DA staining under a fluorescence microscope (magnification, x40). (C) SND protects against $\mathrm{H}_{2} \mathrm{O}_{2}$-induced loss of mitochondrial membrane potential in adrenocortical $\mathrm{H} 295 \mathrm{R}$ cells. Cultured H295R cells were treated as in B and mitochondrial membrane integrity was quantified using JC-1 staining and evaluation under a fluorescence microscope (magnification, $\mathrm{x} 40$ ). Values are expressed as the mean \pm standard error of the mean ( $\mathrm{n}=6 /$ group). ${ }^{\# \#} \mathrm{P}<0.01$ and ${ }^{\# \#} \mathrm{P}<0.001$ vs. blank or $\mathrm{SND}+\mathrm{H} 2 \mathrm{O} 2$ group; ${ }^{* *} \mathrm{P}<0.01$ vs. $\mathrm{H}_{2} \mathrm{O}_{2}$ group. COX-2, cyclooxygenase 2; SND, Sini decoction; ROS, reactive oxygen species; DFCH-DA, 2'-7'-dichlorodihydrofluorescein diacetate.

The present study postulated that, in the context of human KDS-Yang, where circulating ACTH and CORT levels are low and patients present with a dampened immune system, low energy metabolism and altered circadian cycles, activation of these COX-2-driven proliferative and pro-inflammatory effects may be beneficial in restoring the ability of the HPA-axis to respond to physiological stresses. Similarly, despite being a pleiotropic transcription factor, upregulation of $N F-\kappa B$ expression in the adrenal gland in response to SND treatment is also consistent with a stress response role. $N F-\kappa B$ is a rapidly-acting primary transcription factor and has a key role in regulating the immune response to infection (26). Accordingly, SND-mediated activation of NF- $\kappa \mathrm{B}$ expression and activity is likely to restore a level of balance of the HPA-axis and equilibrate the system.

As the present microarray data also indicated a marked anti-oxidant response attributed to SND treatment, the adrenocortical cell line H295R was used for further investigation in vitro. It is worth mentioning that the TCM concept of Yang is closely associated with energy metabolism, and hence, aspects of mitochondrial function were also investigated. Of note, in the context of an external insult $\left(\mathrm{H}_{2} \mathrm{O}_{2}\right.$ incubation), SND treatment exerted a pronounced protective effect by repressing the induction of COX-2 expression. This is in contrast to the microarray data generated using rat adrenal tissue from the KDS-Yang model and suggests that SND treatment has pleiotropic effects, which may be dependent on the cellular context and conditions (i.e. under Yang deficiency, SND appears to provide an activating stimulatory effect through upregulation of COX-2, but when Yang is sufficient but cell-damaging conditions prevail, SND may be protective by downregulating COX-2) (10). This protective effect was further confirmed by prevention of ROS generation and maintenance of the mitochondrial membrane potential in the presence of SND and $\mathrm{H}_{2} \mathrm{O}_{2}$. In the ROS assay, although cells had some fluorescence background as demonstrated in the blank group (Fig. 4B), cells pretreated with SND exhibited effects on the generation of ROS. Indeed, protective effects of SND have also been observed in other disease states, 

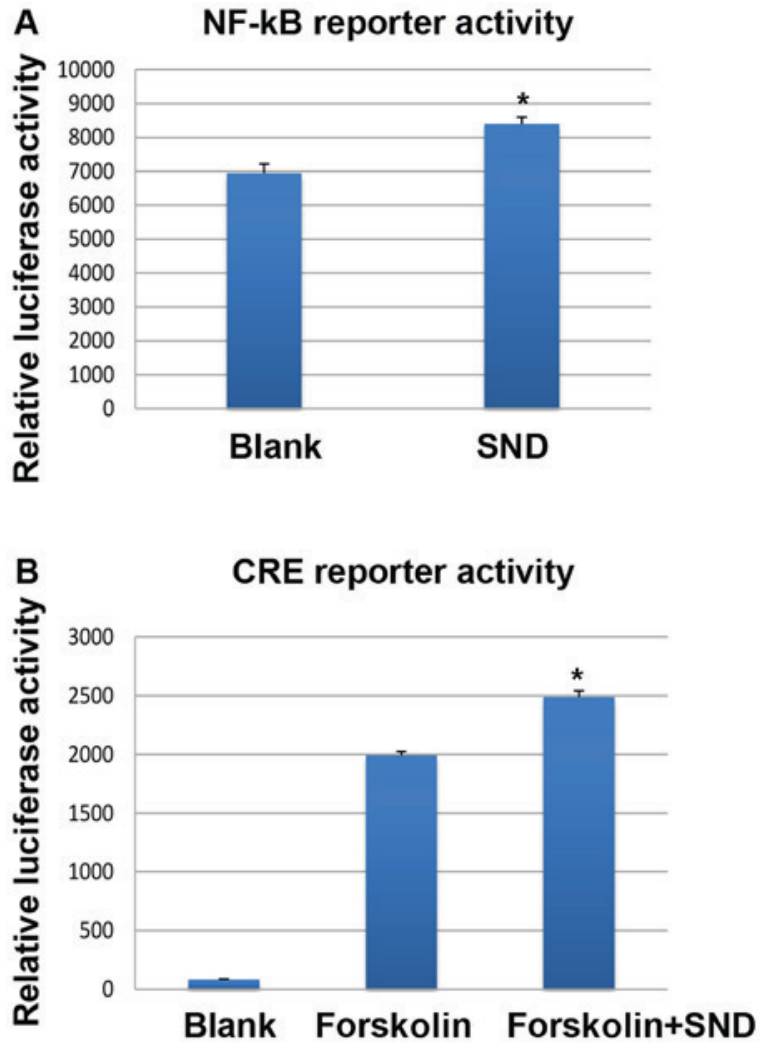

Figure 5. SND treatment upregulates NF- $\mathrm{BB}$ or CRE reporter activity in H295R cells. Adrenocortical H295R cells were stably transfected with luciferase-expressing reporter constructs responsive to either (A) NF- $\mathrm{kB}$ activity or (B) CRE binding protein activity. Luciferase activity was assessed following a 24-h treatment with SND. Values are expressed as the mean \pm standard error of the mean ( $n=6 /$ group). "P $<0.01$ vs. blank or Forskolin group. SND, Sini decoction; NF, nuclear factor; CRE, cyclic adenosine monophosphate response element.

particularly cardiomyocytes in response to oxidative and adriamycin-induced damage $(27,28)$.

The present study further performed a reporter gene induction study using NF- $\mathrm{kB}$ and CRE-responsive constructs for several reasons. First, the microarray data highlighted marked elevations in the expression of these two genes upon SND treatment in the rat adrenal glands. Furthermore, according to DNA sequence analysis, the COX-2 gene promoter contains binding sites for the CRE transcription factor and finally, NF- $\kappa \mathrm{B}$ and cAMP/cGMP have been associated with the concept of Yin and Yang in TCM (9). Indeed, Shen (29) has previously reported downregulation of NF- $\mathrm{kB}$ expression in lymphocytes from a rat model of Yang deficiency, whilst Goldberg et al (30), proposed a control mechanism for opposing cAMP/cGMP in cellular regulation in the 1970 's. The present results indicated that SND treatment was able to stimulate NF- $\kappa B$ and CRE-reporter activity in vitro, thus suggesting that the expression of COX-2 is driven, at least in part, through activation of these transcriptional pathways. However, it is likely that the variety of pharmacologically active components within SND acts via multiple pathways contributing to the gene expression changes observed. Additional studies are currently underway to further elucidate these mechanisms. However, the utilization of animals was a limitation of the present study as individual differences in hormone levels may result in variations in data (Fig. 1D). Methods to prevent these differences should be implemented in future studies.

Taken together, the results of the present study confirmed that SND alleviates the hallmarks of KDS-Yang in a rat model, through restoration of circulating and in situ expression of HPA-axis hormones. Importantly, the characterization of SND-induced gene expression changes in the rat adrenal glands indicated the upregulation of metabolic and stress response-associated signaling pathways, including those involving COX-2 and NF- $\kappa$ B. Further in vitro analyses indicated the protective effect of SND treatment within mitochondria and suggested that the pleiotropic effects of SND are mediated, at least in part, through activation of NF- $\mathrm{KB}$ and CRE signaling. These conclusions offer a molecular insight into the well-established effects of SND in alleviating KDS-Yang.

\section{Acknowledgements}

Not applicable.

\section{Funding}

The present study was supported by the National Natural Science Foundation of China (grant nos. 81573938 and 81460720).

\section{Availability of data and materials}

The datasets used and/or analyzed during the current study are available from the corresponding author on reasonable request.

\section{Authors' contributions}

NT and DM designed and directed the experiments. HQ performed the experiments and wrote the manuscript. LL collected the data. WS analyzed the data. All authors read and approved the final manuscript.

\section{Ethics approval and consent to participate}

The protocols were approved by the ethics committee of Guangxi University of Chinese Medicine (Nanning, China).

\section{Patient consent for publication}

Not applicable.

\section{Competing interests}

The authors declare that they have no competing interests.

\section{References}

1. Shen Z: The location of deficiency syndrome of kidney Yang. Chin Med J (Engl) 112: 973-975, 1999.

2. Liu X, Du J, Cai J, Liu X, Xu G, Lin A and Teng Q: Clinical systematic observation of kangxin capsule curing vascular dementia of senile kidney deficiency and blood stagnation type. J Ethnopharmacol 112: 350-355, 2007.

3. Xiong PH, Zhang L, Tian SF, Gu MH and Chen AP: Effect of equiguard in treating patients with shen-yang deficiency syndrome. Chin J Integr Med 14: 225-227, 2008. 
4. Lang JM, Li MZ and Wei AS: Study on objective parameter of syndrome differentiation of diabetic nephropathy. Zhongguo Zhong Xi Yi Jie He Za Zhi 15: 84-86, 1995 (In Chinese).

5. Deng ZZ, He YT and Yu YM: Comparison between two diagnostic methods of computer's mathematic model and clinical diagnosis on TCM syndromes of rheumatoid arthritis. Zhongguo Zhong Xi Yi Jie He Za Zhi 16: 727-729, 1996 (In Chinese).

6. Gu WL, Shi ZX, Yu YX, Wu YW,Lu BW and Hui KK: Distribution characteristics of syndrome types in essential hypertension. Zhong Xi Yi Jie He Xue Bao 8: 842-847, 2010 (In Chinese).

7. Liu Y, Wu WK, Chen C and Duan XF: Effect of delayed preconditioning induced by Sini decoction on myocardial cell apoptosis and its mitochondrial mechanism. Zhongguo Zhong Yao Za Zhi 31: 1269-1272, 2006 (In Chinese)

8. Zhao MQ, Wu WK, Zhao DY, Duan XF and Liu Y: Protective effects of sini decoction on adriamycin-induced heart failure and its mechanism. Zhong Yao Cai 32: 1860-1863, 2009 (In Chinese)

9. Zhao L, Wu H, Qiu M, Sun W, Wei R, Zheng X, Yang Y, Xin X, Zou H, Chen T, et al: Metabolic signatures of kidney yang deficiency syndrome and protective effects of two herbal extracts in rats using GC/TOF MS. Evid Based Complement Alternat Med 2013: 540957, 2013

10. Huang D, Yang J, Lu X, Deng Y, Xiong Z and Li F: An integrated plasma and urinary metabonomic study using UHPLC-MS: Intervention effects of Epimedium koreanum on 'Kidney-Yang Deficiency syndrome' rats. J Pharm Biomed Anal 76: 200-206, 2013.

11. Tan Y, Liu X, Lu C, He X, Li J, Xiao C, Jiang M, Yang J, Zhou K, Zhang Z et al: Metabolic profiling reveals therapeutic biomarkers of processed aconitum carmichaeli debx in treating hydrocortisone induced kidney-yang deficiency syndrome rats. J Ethnopharmacol 152: 585-593, 2014

12. Tan G, Liao W, Dong X, Yang G, Zhu Z, Li W, Chai Y and Lou Z: Metabonomic profiles delineate the effect of traditional Chinese medicine sini decoction on myocardial infarction in rats. PLoS One 7: e34157, 2012

13. National Pharmacopeia Committee of China. Chinese Pharmacopeia 2010 Edition: 650-660, 2010.

14. Munakata S and Hendricks JB: Effect of fixation time and microwave oven heating time on retrieval of the ki-67 antigen from paraffin-embedded tissue. J Histochem Cytochem 41: 1241-1246, 1993.

15. Livak KJ and Schmittgen TD. Analysis of relative gene expression data using real-time quantitative PCR and the 2(-Delta Delta C(T)) method. Methods 25: 402-408, 2001.

16. Schmittgen TD and Livak KJ: Analyzing real-time PCR data by the comparative C(T) method. Nat Protoc 3: 1101-1108, 2008.

17. Verweij CL, Geerts $M$ and Aarden LA: Activation of interleukin-2 gene transcription via the T-cell surface molecule CD28 is mediated through an NF-kB-like response element. J Bio Chem 266: 14179-14189, 1991.
18. Grewal SS, Fass DM, Yao H, Ellig CL, Goodman RH and Stork PJ: Calcium and cAMP signals differentially regulate cAMP-responsive element-binding protein function via a rap1-extracellular signal-regulated kinase pathway. J Biol Chem 275: 34433-34441, 2000.

19. Chun KS and Surh YJ: Signal transduction pathways regulating cyclooxygenase- 2 expression: Potential molecular targerts for chemoprevention. Biochem Pharmacol 68: 1089-1100, 2004.

20. Tanabe $\mathrm{T}$ and Tohnai $\mathrm{N}$ : Cyclooxygenase isozymes and their gene structures and expression. Prostaglandins Other Lipid Mediat 68-69: 95-114, 2002.

21. Sun W, Yin XJ, Tu Y, Wan YG, Liu H and $\mathrm{Hu} \mathrm{H:} \mathrm{Effects}$ and mechanisms of Qifu decoction ameliorating renal tubulointerstitial fibrosis through inhibiting ERK1/2 signaling pathway in unilateral ureteral obstruction rats with Yang deficiency. Zhongguo Zhong Yao Za Zhi 39: 4082-4089, 2014 (In Chinese).

22. Zhang JH, Xin J, Fan LX and Yin H: Intervention effects of Zuoguiwan containing serum on osteoblast through ERK1/2 and Wnt/ $\beta$-catenin signaling pathway in models with kidney-Yang-deficiency, kidney-Yin-deficiency osteoporosis syndromes. Zhongguo Zhong Yao Za Zhi 42: 3983-3989, 2017 (In Chinese).

23. Huang R, Zhang Z, Xu M, Chang X, Qiao Q, Wang L and Meng X: Effect of Sini decoction on function of hypothalamic-pituitary-adrenal axis in patients with sepsis. Zhonghua Wei Zhong Bing Ji Jiu Yi Xue 26: 184-187, 2014 (In Chinese).

24. Hla T and Neilson K: Human cyclooxygenase-2 cDNA. Proc Natl Acad Sci USA 89: 7384-7388, 1992.

25. Jia Z, Zhang Y, Ding G, Heiney KM, Huang S and Zhang A: Role of COX-2/mPGES-1/prostaglandin E2 cascade in kidney injury. Mediators Inflamm 2015: 147894, 2015.

26. Sha WC: Regulation of immune responses by NF-kappa B/Rel transcription factor. J Exp Med 187: 143-146, 1998

27. Zhao M, Wu W, Duan X, Liu Y, Zhao D, Liang T and Luo H: The protective effects of sini decoction on mitochondrial function in adriamycin-induced heart failure rats. Zhong Yao Cai 28: 486-489, 2005 (In Chinese).

28. Nie Y, Wu W, Liu Y, Duan X, Zhao M and Zhao D: Protective effects of sini decoction on cardiomyocytes in oxidative stress damage induced by peroxide. Zhong Yao Cai 28: 395-399, 2005 (In Chinese).

29. Shen ZY: Rule of tonifying the kidney in regulating T lymphocyte apoptosis in syndrome of kidney-yang deficiency-plasticity of gene balance. Zhong Xi Yi Jie He Xue Bao 2: 321-322, 2004 (In Chinese).

30. Goldberg ND, Haddox MK, Nicol SE, Glass DB, Sanford CH, Kuehl FA Jr and Estensen R: Biologic regulation through opposing influences of cyclic GMP and cyclic AMP: The Yin Yang hypothesis. Adv Cyclic Nucleotide Res 5: 307-330, 1975. 